\title{
Intervenciones sobre los genitales femeninos: Entre el bisturí del cirujano plástico y el cuchillo ritual*
}

\section{Female genital interventions: Between the plastic surgeon's scalpel and the ritual knife}

\author{
MariaCaterina La Barbera \\ Instituto de Lengua, Literatura y Antropología. \\ Centro de Ciencias Humanas y Sociales. CSIC. Madrid.
}

\section{RESUMEN}

Mientras se gastan cifras enormes y cada año crecientes en intervenciones quirúrgicas que modifican los órganos sexuales, las intervenciones rituales sobre los genitales femeninos encuentran una oposición política y social cada vez más fuerte. ¿Cuáles son los modelos interpretativos adoptados para definir como estéticas a unas y criminales a las otras? ¿Existe una actitud colonialista implícita en la condena de las intervenciones rituales sobre los genitales femeninos? Este parece ser el caso cuando se examinan conjuntamente los daños para la salud derivados del implante mamario, los significados simbólicos de las intervenciones rituales, la reinvención estratégica de las prácticas tradicionales y el uso del binomio salud/patología como "proceso de control". Este artículo propone cuestionar la idea de que quien se somete al bisturí del cirujano plástico disfruta de libertad y autonomía en una sociedad libre de opresión, mientras que quien se expone al cuchillo ritual es víctima pasiva de las sociedades patriarcales tradicionales.

Palabras clave: Cuerpo femenino, Mutilación genital femenina, Circuncisión femenina, Ablación de clítoris, Cirugía estética, Ritual, Procesos de control.

\section{SUMMARY}

While enormous and growing sums of money are spent each year in genital cosmetic surgeries, ritual female genital interventions increasingly meet strong political and social

* Este trabajo se ha realizado en el marco del proyecto FFI2009-08762. Agradezco a Laura Nader sus comentarios durante mi estancia posdoctoral en el Departamento de Antropología de la Universidad de Berkeley, a Margarita del Olmo su apoyo durante el proceso de elaboración del texto y a Matilde Fernández Montes su meticulosa revisión. Agradezco a Carmen Ortiz García y a los revisores anónimos sus comentarios críticos. 
opposition. Which interpretative models have been adopted to define some interventions as "cosmetic" and the others as "criminal"? Is there a colonialist attitude implicit in banning ritual female genital interventions? This appear to be the case after a joint examination of the health risks associated with the breast implant, the symbolic meanings of the ritual interventions, the strategic reinvention of traditional practices, and the use of the binomy health/pathology as a "controlling process". This paper challenges the assumption that who is exposed to the plastic surgeon's scalpel enjoys freedom and autonomy in an oppression-free society, while who is subjected to the ritual knife is a passive victim of traditional patriarchal societies.

Key words: Female Body, Female Genital Mutilation, Female Circumcision, Excision, Cosmetic Surgery, Ritual, Controlling Process.

\section{INTRODUCCIÓN}

Mientras el número de intervenciones quirúrgicas sobre los órganos sexuales femeninos aumenta anualmente, se promocionan cada vez más numerosas campañas internacionales en contra de las intervenciones rituales sobre los genitales femeninos. ¿Cuáles son los modelos interpretativos adoptados para definir como estéticas a las unas y como crímenes a las otras? Este artículo adopta la perspectiva de la antropología socio-jurídica (Moore 2005: 360) para llevar a cabo un análisis del discurso teórico-político acerca de las intervenciones sobre el cuerpo femenino sean estéticas o rituales. A través del análisis del poder legitimador de la medicina y la función excluyente de la criminalización operada por el derecho penal, se pretende analizar las contradicciones y los conflictos con relación a las representaciones, modelos interpretativos o ideológicos social y culturalmente situados.

Con el fin de abordar esta controvertida cuestión, se propondrá el examen conjunto de los daños para la salud y los significados socio-simbólicos de las intervenciones estéticas y de las rituales. Se analizará cómo las últimas son entendidas por los grupos que las practican a través de sus funciones sociales, las relaciones de género y la construcción de la identidad étnica en que se arraigan. Se pondrá de relieve cómo el intento de acercarse a estas prácticas usando solo categorías médicas y supuestamente neutrales significa hacer invisibles sus inherentes aspectos socio-relacionales, como si estos fueran accesorios. Además, se destacará que el hecho de usar las categorías médicas como si fueran absolutas conduce a ignorar que estas también están culturalmente determinadas. Al respecto, se hará hincapié en su análisis como "procesos de control" a la luz del caso emblemático de la cirugía intersexual.

Este artículo se centrará en las prácticas realizada en la diáspora, refiriéndose en particular a las mujeres migrantes (o hijas de migrantes) prove- 
nientes de aquellos países subsaharianos donde tradicionalmente se realizan las intervenciones rituales, o sea la población a la que se refieren las leyes europeas que prohíben la llamada "mutilación genital femenina" (La Barbera y Cortina 2010) ${ }^{1}$.

Se compararán por lo tanto las intervenciones estéticas y rituales, ambas llevadas a cabo en Europa y EE.UU, unas legítimamente y otras clandestinamente, unas por nacionales, las otras por mujeres migrantes de origen subsahariano. El objetivo es cuestionar el supuesto de que someterse al bisturí del cirujano plástico representa una expresión de libertad y autonomía, mientras que hacerlo al cuchillo ritual es sinónimo de ser víctima pasiva de una sociedad patriarcal tradicional. Al contrario, las prácticas culturales serán examinadas como lugares de posibilidades múltiples, donde los individuos y los grupos las inventan y se reinventan a sí mismos activa y estratégicamente.

\section{INTERVENCIONES ESTÉTICAS SOBRE LOS GENITALES FEMENINOS}

De acuerdo con el informe de 2009 de la Asociación Americana de Cirujanos Plásticos, se gastan anualmente diez millardos de dólares en intervenciones estéticas ${ }^{2}$. Por razones estéticas, se realizan cada vez más modifi-

${ }^{1}$ Véase la siguiente tabla con los datos elaborados en La Barbera y Cortina (2010).

\begin{tabular}{|c|c|c|c|c|c|c|c|c|}
\hline \multirow{4}{*}{ Población total } & \multicolumn{8}{|c|}{ Población extranjera por edad, sexo y país de origen (2006-2009) } \\
\hline & \multirow{2}{*}{\multicolumn{2}{|c|}{$\begin{array}{c}\text { Francia } \\
63.186 .098 \\
\end{array}$}} & \multirow{2}{*}{\multicolumn{2}{|c|}{$\begin{array}{c}\text { Reino Unido* } \\
53.912 .000 \\
\end{array}$}} & \multirow{2}{*}{\multicolumn{2}{|c|}{$\begin{array}{c}\text { Italia } \\
60.045 .068 \\
\end{array}$}} & \multirow{2}{*}{\multicolumn{2}{|c|}{$\begin{array}{c}\text { España }{ }^{* * *} \\
46.745 .807 \\
\end{array}$}} \\
\hline & & & & & & & & \\
\hline & $\%$ & Sex ratio & $\%$ & Sex ratio & $\%$ & Sex ratio & $\%$ & Sex ratio \\
\hline Población extranjera & 5,8 & 1,1 & 12,5 & - & 6,5 & 1,0 & 12,1 & 1,1 \\
\hline Población subsahariana & 12,2 & 1,1 & 10,9 & 1,0 & 8,8 & 1,6 & 3,9 & 3,0 \\
\hline Tipo I e II*** & 97,6 & - ***** & 78,1 & - **** & 66,3 & 1,3 & 61,7 & 2,7 \\
\hline Tipo II e III**** & 2,4 & — **** & 21,9 & - **** & 31,4 & 2,7 & 32,1 & 5,8 \\
\hline Menores subsharianas & 25,6 & 1,0 & 30,8 & 1,1 & - & - & 9,3 & 1,6 \\
\hline
\end{tabular}

FuENTES: Francia (Censo de la población 2006); Reino Unido (calculo estimado 2008/2009); Italia (Población residente a 31 diciembre de 2008); España (Registro de la población a 1 enero de 2009).

* Reino Unido clasifica la población extranjera en base a el origen étnico y no la nacionalidad como los demás países.

** La prevalencia de la población subsahariana en España se concentra en Catalunya.

*** La clasificación de la población de origen subsahariana se basa en el tipo de intervención ritual sobre los genitales femeninos practicado en los países de origen. Tipo I= Incisión de la capucha clitoridea. Tipo II= Remoción del clítoris. Tipo III= Sutura de los labios mayores .

**** Para Reino Unido y Francia la fuente es Guiné y Moreno Fuente (2007).

${ }^{2}$ Véase "2010 Report of the 2009 Statistics National Clearinghouse of Plastic Surgery Statistics", http://www.plasticsurgery.org/Documents/Media/statistics/2009-US-cosmeti creconstructiveplasticsurgeryminimally-invasive-statistics.pdf [última consulta en fecha 01/ 05/2010]. 
caciones quirúrgicas de los genitales femeninos, incluyendo operaciones que prevén la reparación del himen, el estrechamiento vaginal, la eliminación de la capucha del clítoris y la reducción del tamaño de los labios. En particular, el rejuvenecimiento vaginal láser (RVL) es utilizado para estrechar la vagina y mejorar la satisfacción sexual, mientras que la vaginoplastia láser se emplea para modificar el tamaño y la forma de labios y capucha clitorídea. El Dr. Matlock es conocido en todo el mundo por realizar rejuvenecimiento vaginal, vaginoplastia, aumento del G-spot y levantamiento de glúteos en el Laser Vagina Rejuvenation Institute of Los Angeles ${ }^{3}$.

Todavía más frecuente es la mamoplastia de aumento (con implantes de silicona o salinos). El esperado aumento del sex-appeal, la supuesta consecuente mejora de la autoestima y del reconocimiento social, motiva a muchas chicas adolescentes y mujeres adultas a someterse a estas intervenciones. Además, la Sociedad Americana de Cirujanos Plásticos ha definido el hecho de tener pechos pequeños como una enfermedad grave que comporta sentimientos de inadecuación, falta de confianza en sí misma y problemas en la percepción de la propia feminidad y bienestar (Coco 1994: 104110). Hablando desde la altura del templo de la "Medicina", los cirujanos plásticos alcanzan un enorme poder persuasivo y una indiscutible autoridad desde la cual definen lo que es patología, enfermedad o deformación. A la "patología" de tener los pechos pequeños se le ha dado el nombre de micromastia y se le ha encontrado una "cura" que constituye un negocio multimillonario en EE.UU y Europa. Cada implante de mama cuesta entre 3.000 y 4.000 dólares y solo en los Estados Unidos se han realizado una media de 215.000 intervenciones anuales en los últimos quince años, con un aumento estimado del $36 \%$ en la última década ${ }^{4}$.

Sin embargo, esta "cura" tiene contraindicaciones para la salud a corto y largo plazo. Las complicaciones a corto plazo son esencialmente las que derivan de cualquier intervención quirúrgica, o sea hemorragias, infecciones y hematomas. Mientras que las a largo plazo van desde las dificultades en detectar formaciones cancerosas a través de la mamografía, formación de queloides y contracturas capsulares (casi en el 60\% de las pacientes), hasta la atrofia muscular de la zona mamaria, los trastornos auto-inmunes y la pérdida de sensibilidad en los pezones ${ }^{5}$. Además, la deflación del implante

\footnotetext{
3 Véase http://www.drmatlock.com/gallery.htm [última consulta en fecha 01/05/2010].

${ }^{4}$ Véanse las estadísticas de la National Clearinghouse of Plastic Surgery Statistics, http:/ /www.plasticsurgery.org/Media/Statistics.html [última consulta en fecha 01/05/2010].

5 William Master y Virginia Johnson advirtieron la imposibilidad de destacar distinciones fisiológicas entre orgasmos procedentes de la estimulación del clítoris o del pezón mamario (Masters et al. 1995: 84). Es significativo, sin embargo, que la pérdida de
} 
y los escapes de silicona, que con el tiempo se producen inevitablemente, requieren nuevas cirugías con las consiguientes complicaciones relacionadas. Junto con estas deflaciones del implante se dan también problemas de salud mental (Coco 1994: 126). A pesar de todo esto, sin embargo, la implantación de mama está permitida en todos los países occidentales, incluso en menores de edad con el consentimiento de solo uno de los padres ${ }^{6}$.

Es debatido si la implantación de mama aspira a reescribir la geografía del cuerpo de acuerdo con un ideal patriarcal internalizado de la feminidad (Coco 1994), o si más bien se trata de una forma extrema de empoderamiento de las mujeres a través de la disposición sobre su cuerpo, pudiendo modelarlo a su gusto y placer ${ }^{7}$. Si algunas autoras argumentan que los implantes mamarios, así como la vaginoplastia estética, revelan el deseo interiorizado de las mujeres de ajustarse a una imagen corporal ideal, creada por las fantasías sexuales de los hombres; otras argumentan que el feminismo es un movimiento que revindica el derecho de las mujeres a controlar sus vidas y sus cuerpos cualquiera que sea su elección ${ }^{8}$.

sensibilidad en los pezones y el consiguiente obstáculo al funcionamiento sexual satisfactorio no sea considerado de ninguna manera "mutilante".

${ }^{6}$ Véase http://www.plasticsurgery.org/Media/Briefing_Papers/Plastic_Surgery_for_ Teenagers.html. [última consulta en fecha 01/05/2010].

7 "Cosmetic surgery is an intervention in a person's identity - that is, as a response to suffering evoked by having a body that is experienced as too 'different' or 'abnormal' to be endured. Treating it exclusively in terms of beauty does justice neither to the gravity of the person's suffering nor to the moral implications of cosmetic surgery as an imperfect but, in some cases, defensible remedy for this suffering. It seems to me that this perspective should - in principle - be applied to any person who under- goes cosmetic surgery. In other words, all recipients of cosmetic surgery should be regarded as negotiating their identities in a context where differences in embodiment can evoke unbearable suffering [...]. While I would argue that cosmetic surgery is best seen as an intervention in identity for everyone, regardless of gender or ethnicity, this does not entail that all cosmetic surgeries have the same meaning. Identities are negotiated in specific historical and social contexts in which cultural constructions of race, ethnicity, gender, sexuality, age and nationality shape how individuals perceive their bodies as well as the kinds of bodily practices that are considered desirable, acceptable or appropriate for altering the body" (Davis 2003: 84).

8 "Although feminist scholars have universally attacked oppressive sexual and beauty standards, there is more than one feminist position when it comes to surgery. Does cosmetic surgery offer nothing but harm to women or can it be beneficial, depending on political and social context? Analogous arguments are seen on other contentious topics for feminists such as sex work and pornography, and even the medicalization of sexuality. Is there any way for women to find value in these spheres or are they nothing but arenas for exploitation and oppression? Most feminists feel that the women's movement was all about women winning the right to control their lives (and bodies) howsoever they 
No obstante, sea o no implícita la estructura patriarcal en el implante de mama y en la vaginoplastia estética, estas intervenciones no parecen a los legisladores occidentales tan problemáticas como para prohibirlas. No importa la manera en que las elecciones de las mujeres se hayan formado, cuando se solicitan estas cirugías se asume que las personas son libres y autónomas. Y desde luego sería una invasión extremadamente paternalista evaluar — admitiendo que esto fuera posible - en qué manera se ha formado el consentimiento y las preferencias de una mujer adulta 9 .

\section{EL PODER DE DAR NOMBRES}

Como dice un proverbio nigeriano, hasta que el león no cuente su historia, los cuentos sobre la caza solo celebrarán a los cazadores. Awa Thiam en su ensayo La Parole aux négresses alega no solo la necesidad de luchar contra las costumbres opresivas para las mujeres, sino también de que ellas hablen por sí mismas. Los "occidentales" deberían tomar consciencia de que su juicio se basa en criterios que no guardan ninguna relación con la mentalidad de los pueblos sometidos a su consideración y que atacan a los africanos de manera análoga a como lo hizo el colonialismo (Thiam 1986: 80).

Además, no se puede subestimar el efecto que produce cambiar el nombre de las prácticas culturales en aquellas culturas que conceden una importancia tan profunda a los nombres que se celebran ceremonias para establecerlos. Sin embargo, los "occidentales" nos apresuramos a apropiarnos del poder de nombrar, mostrándonos totalmente insensibles a sus implicaciones y consecuencias. Desde luego, la cuestión de nombrar se refiere a un eterno dilema de la antropología: “¿cómo puede el antropólogo ser capaz de interpretar los símbolos rituales de una sociedad más profunda y comprensivamente que los propios actores?" (Turner 1980: 29). Dicha cuestión además de revelar la diferencia entre la perspectiva del participante y la del observante, implica la cuestión del poder de dar nombres, siendo reveladora de la posición de hegemonía epistemológica que es el tema central de la teoría postcolonial (Spivack 1988).

Los teóricos políticos occidentales utilizan comúnmente el término "mu-

chose. There are, broadly speaking, two positions on choice in the feminist essays and books examining cosmetic surgery as a gendered practice. Those in the first group feel that the issue of "choice" regarding cosmetic surgery is fraught because the context within which women make their decisions is filled with misogynist influences" (Tiefer 2008: 473).

9 Véase Chamber (2004), Meyer (2000) y Nussbaum (1999: 118-129). 
tilación genital femenina" ${ }^{10}$ Por "mutilación" se entiende lisiar o cortar una extremidad o un órgano. Su uso implica un inequívoco juicio de valor. Desde la perspectiva occidental, estas prácticas son solo causa de enfermedades, irreparable desfiguración del cuerpo y privación permanente de la integridad física. Lo que aquí subyace es la consideración de las culturas africanas y musulmana como barbáricas, y la importancia —e incluso indispensabilidad- de Occidente para purgar dicha barbarie. Obioma Nnaemeka lamenta que solo la arrogancia imperialista puede imaginar lo que los africanos quieren, determinar lo que necesitan y encontrar formas de entregarles los bienes supuestamente necesitados, marcando una nueva época de colonialismo y celo misionero (Nnaemeka 2001: 178). Esta perspectiva ignora las cuestiones de identidad, cultura y normas sociales que están entrelazadas en estas prácticas. Ignora además que las poblaciones que las practican no las perciben como mutilación, sino más bien como modificaciones de los órganos genitales realizadas para satisfacer cánones de belleza, higiene y orden social —en el que se inserta la construcción del género- profundamente arraigados en su cultura" ${ }^{11}$ Ya que el término "mutilación genital femenina" implica un juicio de valor, considero improductivo adoptarlo en el análisis de dichas prácticas que llevaré a cabo en este artículo ${ }^{12}$.

A su vez, las comunidades que practican estos rituales utilizan la expresión "circuncisión femenina" en lugar de "mutilación", rechazando así la idea de que sus mujeres estén desfiguradas y que, a su vez, ellas estén desfigurando a sus hijas. Utilizando el término "circuncisión", hacen hincapié en la dimensión inherentemente iniciática de estas prácticas, a las que se someten todos, chicos y chicas, para entrar en la edad adulta. La expresión "circuncisión femenina" muestra además el paralelismo que existe entre las intervenciones sobre los genitales masculinos y femeninos dentro de las comunidades que practican estos rituales ${ }^{13}$. Sin embargo, esta analogía no

${ }^{10}$ En España se usa mayoritariamente el término "ablación de clítoris" como término general para hacer referencia a los distintos tipos de intervenciones rituales, a pesar de que no todos prevén la remoción del órgano.

11 Anexo a "USAID Policy on Female Genital Cutting (FGC): Explanation of Terminology", en http://www.usaid.gov/our_work/global_health/pop/techareas/fgc/annex.html [última consulta en fecha 01/05/2010].

12 Maria Luisa Ciminelli comenta: "L'adozione acritica dell'etichetta lingüististica Mgf sembra in realtá comportare il rischio, per l'antropologia, di svolgere ancora una volta nei confronti della medicina quel ruolo subalterno e ancillare che dimostra (e deriva da) una scarsa consapevolezza epistemologica della propia autonomia disciplinare" (Ciminelli 2002: 43).

13 En todas las sociedades donde tradicionalmente se realizan las intervenciones rituales sobre los genitales femeninos también se práctica la circuncisión masculina. Sin 
se da en el caso de las intervenciones más invasivas como la escisión y la infibulación. Por esta razón, la expresión "circuncisión" me parece inadecuada para describir la completa variedad de las intervenciones realizadas.

Aunque ningún nombre tenga valor neutral en sí mismo, un intento exitoso de nombrar estas prácticas se llevó a cabo en 1996 por el United Nations Population Fund (UNPFA) con la expresión "female genital cutting" (FGC), que podríamos traducir como "corte genital femenino". Esta decisión fue motivada por el rechazo de la expresión "mutilación genital femenina" por parte de muchos activistas y comunidades que lo consideran no propicio para el debate y la colaboración. Dado que la intención explícita fue la de no expresar juicios de valor y evitar la demonización de algunas culturas, la expresión "corte genital femenino" ha sido adoptada en los documentos oficiales más recientes de la United States Agency for International Development (USAID) ${ }^{14}$. Sin embargo, el término no se ha difundido en los países de habla no inglesa, ya que usar la palabra "corte" en relación al cuerpo sigue siendo perturbador (Obermeyer 2005: 444).

Es preciso recordar, en fin, que con el objeto de evitar una perspectiva etnocéntrica, algunas antropólogas han adoptado las expresiones "operación genital femenina" (Walley 1997), "cirugía genital femenina” (Obermeyer 1999), "modificaciones de los genitales femeninos" (Fusaschi 2003), "modificación corporal" (El Safti 2007). Aunque estas apunten eficazmente a la idea de alteración corporal, es cierto que no permiten marcar una diferencia suficientemente clara entre las intervenciones rituales prohibidas por las leyes occidentales (y realizadas clandestinamente) y las de tipo estético, modificadoras del cuerpo femenino, que se realizan legalmente en clínicas y hospitales.

embargo, en los países occidentales, mientras la circuncisión masculina está permitida y es corrientemente practicada en los hospitales, las intervenciones rituales sobre los genitales femeninos están prohibidas y perseguidas como crímenes. Según la National Organization to Halt the Abuse and Routine Mutilation of Males (http://noharmm.org/ HGMstats.htm, última consulta en fecha 01/05/2010), la circuncisión masculina es practicada en EE.UU de forma rutinaria en el $60 \%$ de los recién nacidos. La razón de tal difusión es permitir una higiene más simple por parte de la madre, evitando contactos con el pene considerados impropios (Van Howe 1997). Véase el informe del año 1999 de la Medical American Asociation (AMA), http://www.ama-assn.org/ama/no-index/aboutama/13585.shtml [última consulta en fecha 01/05/2010].

${ }^{14}$ Anexo a "USAID Policy on Female Genital Cutting (FGC): Explanation of Terminology", en http://www.usaid.gov/our_work/global_health/pop/techareas/fgc/annex.html [última consulta en fecha 01/05/2010]. 


\section{UN INTENTO DE APROXIMACIÓN}

Teniendo en cuenta todas estas razones, adopto aquí la expresión "intervenciones rituales sobre los genitales femeninos". Por intervenciones rituales sobre los genitales femeninos se entiende aquí circuncisión (tipo I), escisión (tipo II) e infibulación (tipo III) ${ }^{15}$. La circuncisión, también llamada "clitoridotomía"16, es la forma más suave de corte genital e implica la incisión de la capucha clitorídea. A pesar de no ser un requerimiento islámico, en el mundo musulmán la circuncisión se conoce como "sunna" (tradición), ya que se menciona en algunos ahadith (los dichos del profeta Mahoma) ${ }^{17}$. Este tipo de intervención es el equivalente de la circuncisión masculina. A su vez, la escisión, también conocida como clitoridectomía ${ }^{18}$, implica la re-

${ }^{15}$ La Organización Mundial de la Salud distingue entre tipo I, II y III, http:// www.unfpa.org/webdav/site/global/shared/documents/publications/2008/eliminating fgm.pdf [última consulta en fecha 01/05/2010]. Cabe notar en fin que la OMS ha identificado un cuarto grupo de intervenciones: "Type IV: Unclassified. All other harmful procedures to the female genitalia for non-medical purposes, for example, pricking, piercing, incising, scraping and cauterization". A pesar de que la "circuncisión simbólica" no prevé de ninguna manera una remoción del tejido, esta práctica de "iniciación sin corte" ha sido incluida entre las "mutilaciones" sufridas por las mujeres africanas. Véase La Barbera (2009: 486).

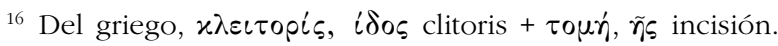

${ }^{17}$ Los ahadith generalmente relacionados con la circuncisión femenina son: "Touch but not destroy. It is more illuminating to the woman and more enjoyable to the husband"; "Do not go deep. This is enjoyable to the woman and preferable to the husband"; "Circumcision is sunnah (recommended) for the men and makrumab (meritorious) for the women" (citados en El-Tom 1998: 165). Aunque las intervenciones rituales sobre los genitales femeninos no estén contempladas en ninguna de las tres religiones monoteístas, a menudo se consideran un requisito religioso, sobre todo por muchos musulmanes creyentes. Sin embargo, contrariamente a lo que comúnmente se supone, la costumbre no se originó en el Islam (Abu-Sahlieh 1994: 82-85; El Safti 2007: 147). De hecho, en países de mayoría musulmana, como Turquía y Marruecos, estas prácticas no se conocen. Es importante recordar que en África el ritual de los cortes genitales femeninos se realiza entre musulmanes, cristianos, animistas y falashas, los judíos de Etiopía (Abu Sahlieh 1994), confirmando su carácter consuetudinario. Para un análisis del posicionamiento del Cristianismo y del Islam ante las intervenciones rituales sobre los genitales femeninos en Egipto, véase Aixelá (2009).

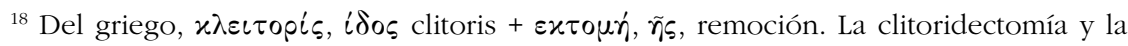
histerectomía —o sea eliminación de ovarios y útero- se practicaron en la Inglaterra victoriana del siglo XIX como soluciones quirúrgicas para curar comportamientos sexuales femeninos considerados anómalos y sintomáticos de trastornos mentales. En concreto, se utilizaba para el tratamiento de la masturbación, de la inclinación lesbiana, de la hipersexualidad y de la histeria. En los EE.UU, hasta el año 1905 hay también constan- 
moción de todo el clítoris y puede incluir el corte de los labios mayores y menores. Por último, la infibulación es la forma de intervención ritual más drástica. Consiste en la sutura de los labios mayores de manera que solo quede un pequeño orificio para la orina y el flujo menstrual y puede conllevar la eliminación completa del clítoris y de los labios menores ${ }^{19}$. Este ritual también se conoce como "circuncisión faraónica", ya que el estudio de algunas momias ha revelado que se realizaba en el antiguo Egipto (Wasunna 2000: 104).

Se ha adoptado aquí la expresión "intervenciones rituales sobre los genitales femeninos" por varias razones. En primer lugar el término "intervención" es utilizable también con referencia a las intervenciones quirúrgicas y estéticas, permitiendo establecer un paralelismo entre todas. Además, la forma plural sugiere la variedad incluida bajo esta denominación. Por último, el adjetivo "ritual" alude a la función socio-simbólica que estas prácticas desempeñan ${ }^{20}$.

En esta línea, cabe subrayar que considero elementos fundamentales para definir un ritual social la dimensión grupal o colectiva, la representación simbólica de momentos clave de la vida individual y social (reiteración de vínculos sociales, momentos de conflicto o cambio de estatus) y la más o menos invariada secuencia ceremonial.

Como actos colectivos, los rituales pueden conllevar la creación de vínculos interpersonales, un sentido de unidad y armonía del grupo. Esta es una experiencia común, por ejemplo, en los conciertos de rock, donde el público arrastrado por el ritmo de la música parece llenarse de energía compartida y casi convertirse en una parte orgánica de un todo que pulsa (Hart, Stevens y Lieberman 1990: 144). Según la mayoría de las teorías, el ritual requiere la repetición grosso modo idéntica de actos de la vida diaria que

cia de casos en los que se cosían juntos los labios vaginales —es decir, se infibulabanpara prevenir la masturbación (Sheehan 1981). Además, hasta el año 1935 se utilizó la clitoridectomía en los hospitales psiquiátricos para tratar la epilepsia, la catalepsia, la melancolía e incluso la cleptomanía. Es interesante observar que en 1925 Sigmund Freud afirmaba que la "eliminación de la sexualidad clitorídea es una condición necesaria para el desarrollo de la feminidad" (Freud 1925: 255).

19 Derivado del latín "fibula" (pinza), el término recuerda el pasador utilizado no sólo para mantener cerrada la toga romana sino también para "cerrar" los genitales de los esclavos e impedirles las relaciones sexuales. Véase Eliminating Female Genital Mutilation: An Interagency Statement, http://www.unfpa.org/webdav/site/global/shared/ documents/publications/2008/eliminating_fgm.pdf [última consulta en fecha 01/05/2010].

${ }^{20}$ Pia Grassivaro Gallo (2006) sugiere interesantemente la expresión "modificazione etniche dei genitali femminili". Sin embargo, el termino "étnico" marca una otredad que el termino "ritual" no implica desde el punto de vista antropológico. 
adquieren un significado ulterior gracias a su referencia a un horizonte simbólico (Kertzer 1988: 9). Piénsese, por ejemplo, en el acto de comer el pan durante la celebración de la eucaristía en el rito litúrgico católico (Mitchel 1996: 490). A través de la repetición rítmica, el estilo evocador y la estimulación sensorial (trajes, música, incienso, etc.), los rituales colectivos generan emociones intensas que, a su vez, fundamentan creencias (Rappaport 1999: 24).

Gracias a estas características, el ritual cumple una función de "estabilización cognitiva" y vela el desorden que subyace en cada cultura (Moore y Myerhoff 1977: 17). El nacimiento, el paso a la adolescencia, a la edad adulta (primera comunión, graduación, la boda) y la muerte; la formación de la nación, la independencia, la liberación nacional: todos ellos son ejemplos de momentos de cambio crucial en la vida del individuo o del grupo. Ya que todo cambio produce estrés, el ritual juega un papel fundamental proveyendo un marco simbólico de referencia y de estabilización cognitiva (Bell 1997; Davis-Floyd 2008). Además, a través de la representación del sistema de creencias de una cultura, el ritual es funcional para la preservación y transmisión de ese mismo sistema de creencias, convirtiéndose en una fuerza importante de la conservación del statu quo. Normalmente quien está en el poder aspira a mantener el control sobre los rituales, utilizando su tremenda fuerza para reforzar, tanto sus propios privilegios, como la importancia de las creencias y sistemas de valores que le mantienen en el poder (DavisFloyd: 2008). Sin embargo, se ha subrayado la potencial divergencia entre las diferentes interpretaciones de un ritual por parte de los distintos participantes, ya que los símbolos pueden ser leídos de diferentes maneras dependiendo de los puntos de vista. La inherente multiplicidad semántica de los símbolos justifica las divergencias interpretativas y explica la posibilidad de mantener el ritual y contextualmente modificarlo tanto en su meta-discurso como en su estructura.

El ritual, como parte de la estructura social, no solo la representa, sino que actúa sobre e interactúa con ella. Desde esta perspectiva, el ritual constituye una poderosa forma de trasformación social. A pesar de toda su insistencia en la continuidad y el orden, el ritual puede terminar siendo un factor importante no solo en la transformación individual, sino también en el cambio social. De hecho, los rituales representan uno de los mayores potenciales de transformación social ya que proporcionan una revitalización de la tradición en respuesta a las cambiantes circunstancias sociales. El estudio de las múltiples potencialidades de los rituales es fundamental para imaginar las perspectivas de cambio socio-político (Kelly y Kaplan 1990: 141). 


\section{INTERVENCIONES RITUALES SOBRE LOS GENITALES FEMENINOS}

Numerosos estudios analizan los significados simbólicos, así como sus vínculos con las relaciones sociales, la percepción estética del cuerpo y las creencias religiosas de las intervenciones rituales sobre los genitales femeninos $^{21}$. La definición de la edad, la identidad étnica, el género y el estatus social explican el papel socio-simbólico desempeñado por estos rituales que los "occidentales" minusvaloran sin considerarlo seriamente. Sin embargo, estas explicaciones que pueden ser etiquetadas como ingenuas desde el punto de vista de un observador occidental podrán, al contrario, ser de gran ayuda en la comprensión de estas prácticas, ya que reflejan aspectos diferentes, y a su vez todos relevantes, de estas intervenciones rituales.

Las intervenciones rituales sobre los genitales femeninos han sido interpretadas como ritos de iniciación que marcan el paso de la pubertad a la edad adulta y en algunos casos constituyen el requisito para formar parte de la sociedad secreta de las mujeres ${ }^{22}$. Una característica importante y finalidad general de las sociedad secretas, de hombres y mujeres, es el sentido de camaradería que generan. Los vínculos comunes de la sociedad unen a los hombres con los hombres y a las mujeres con las mujeres, como miembros de una red más amplia, de una manera que trasciende todas las barreras de la familia, el clan, la tribu y la religión. Este sentido de pertenencia - que surge en gran parte de la memoria de experiencias compartidas en temprana edad- es el factor de mayor importancia en las sociedades secretas (Little 1949: 5).

Los rituales de entrada constituyen tradicionalmente un motivo de celebración y son acompañados con alimentos especiales, danzas y canciones ${ }^{23}$.

${ }^{21}$ El origen de estos rituales no es bien conocido. No hay pruebas concluyentes que aclaren cuándo y dónde se inició la costumbre ni cómo se propagó. Como se practican en muchos países africanos, en parte de la península arábiga, en algunas zonas de Oriente Medio y también entre algunos comunidades en América del Sur, India, Indonesia y Malasia, no hay consenso sobre si se originaron de forma autónoma en las diferentes áreas o si se han propagado a partir de una sola localidad.

22 Si bien son generalmente realizados con motivo de la menarquía, algunos grupos practican las intervenciones rituales sobre los genitales femeninos pocos días después del nacimiento. Por esta razón algunos estudiosos rechazan la hipótesis de que se trate de un ritual iniciático para entrar en la edad adulta. Sobre las tendencias actuales de reducir la edad de las niñas y cambiar el ritual tradicional por un ambiente hospitalizado más seguro, véase Hernlund (2000) y Shell-Duncan (2001).

${ }^{23}$ Véanse los versos que las iniciadas cantan cuando van hacia la casa donde se practica el ritual: "Chicas circuncisas, vamos, vamos a casa. Habéis llegado como chicas incircuncisas y ahora volvéis como mujeres. Chicas circuncisas, vamos, vamos a ir a casa a comer una cabra con nkobe [queso] y nadie os molestará. No entréis en la casa 
Durante la iniciación se les enseña higiene, normas sociales y secretos de la sabiduría tradicional útiles para la salud femenina. El proceso de iniciación incluye la intervención ritual (sin anestésico) sobre los genitales.

El dolor es parte integrante del pasaje de la infancia a la edad adulta. Al dolor, como experiencia intensa e inolvidable, se le reconoce un papel funcional en la transformación de niñas y niños en mujeres y hombres. El ritual iniciático desempeña en este sentido un papel crucial en la creación de las relaciones sociales. Las niñas iniciadas en una misma ceremonia comparten la misma experiencia y desarrollan un fuerte sentido de solidaridad, mutua ayuda y hermandad (Kenyatta 1938: 2; Morinis 1985: 164).

El ritual se realiza con el propósito de purificar, higienizar y embelle$\operatorname{cer}^{24}$. Por costumbre se realiza exclusivamente entre mujeres. El ritual es la forma de transferir la cultura femenina de una generación a la siguiente (Althaus 1997: 132). Esta es una de las razones, explica Obioma Nnaemeka, por la que las comunidades oponen fuerte resistencia al esfuerzo de erradicar estas prácticas (Nnaemeka 2001: 180-181).

Como parte del rito de iniciación a la edad adulta, los adolescentes tienen que perder los símbolos de su dualidad sexual para asumir los órganos y las funciones de adultos (Blackledge 2005: 137-140). Las intervenciones rituales sobre los genitales permiten definir socialmente el sexo de la niña y afirmar su identidad de género (Shweder 2002; Gruenbaum 2001; Grande 2004). La función de la definición del género desempeñada por las intervenciones rituales sobre los genitales femeninos está estrictamente relacionada con la posibilidad de casarse. Una chica que no haya sido sometida a la intervención ritual es considerada inaceptable para el matrimonio. El dolor causado por estas intervenciones realizadas sin el uso de anestésicos es considerado a menudo preferible al ostracismo social que derivaría de lo contrario.

Aunque realmente no sean garantía de virginidad, ya que una nueva intervención antes de la boda puede "restaurarla", las intervenciones rituales sobre los genitales femeninos de tipo III (infibulación) marcan simbólicamente la virginidad, con el fin de celebrar la fertilidad y subrayar el papel de la mujer como madre. Entre los Dogón de Mali se cree por ejemplo

[de los padres]. Que los padres digan, [el clítoris] ha sido eliminado. Dejadla entrar y casarse" (Thomas 2003: 32, traducción propia).

${ }^{24}$ En Egipto, por ejemplo, una chica que no ha sido sometida a la intervención ritual sobre los genitales es llamada "nigsa" (sucia), y en Sudán el término coloquial para la infibulación es "tahúr" (limpieza), depuración. También en Malí y Mauritania, el clítoris se considera feo y el corte se conoce como "tizian" (hacer más bello) y "gaad" (cortar). (Véase Erlich 1986: 193). 
que el clítoris afecta a la fertilidad ya que puede matar al hombre durante las relaciones sexuales o dañar a los niños al parir (Blackledge 2005: 137140). La circuncisión como acto simbólico se enfoca marcadamente hacia la fertilidad potencial, minimizando dramáticamente la sexualidad inherente. Al insistir en la circuncisión de sus hijas, las mujeres afirman su posición social indispensable, una importancia que no consiste en ser las parejas sexuales de sus maridos, sino las madres de los hombres. En esta sociedad las mujeres no logran el reconocimiento social por ser iguales a los hombres, sino por ser cada vez menos parecidas a ellos desde el punto de vista físico, sexual y social. Sendos ritos de circuncisión masculina y femenina destacan esta complementariedad (Boddy 1982: 687).

\section{LA REINVENCIÓN ESTRATÉGICA DE LAS PRÁCTICAS CULTURALES}

El significado de las intervenciones rituales sobre los genitales femeninos está profundamente entrelazado con la identidad étnica y la estructura social centrada en el grupo (Grande 2004: 9). Junto con las cicatrices faciales, los tatuajes, los piercings, las indumentarias, las lenguas y las religiones, las diferencias en el tipo de intervención genital funcionan como marcadores étnicos. Las intervenciones rituales sobre los genitales femeninos constituyen elementos fundamentales para la psicología tribal, ya que recuerdan a sus miembros su pertenencia al grupo (Kenyatta 1938: 133). Desde esta perspectiva, es posible entender por qué las leyes que prohibieron las intervenciones rituales sobre los genitales femeninos durante la ocupación colonial fueron recibidas por muchos africanos como una amenaza a su identidad étnica y una injerencia en su cultura y orden social. Esto explica también el rol que han que desempeñado las intervenciones sobre los genitales femeninos en la resistencia contra el poder colonial y que se hayan convertido en símbolos de identidad africana y de liberación ${ }^{25}$.

A propósito, es emblemática la historia keniana de Ngaitana, que significa "voy a circuncidarme a mí misma". En 1956, durante la ocupación co-

${ }^{25}$ El Reino Unido tiene una larga historia de legislación colonial con la que ha intentado en vano erradicar las intervenciones rituales sobre los genitales femeninos en África. Numerosos misioneros llevaron a cabo campañas de evangelización y abandono de estas prácticas tradicionales y muchas feministas se unieron, ayer como hoy, en la misión. Como señala Susan Pedersen, mientras feministas británicas iban buscado la emancipación política afirmando que los intereses de la mujer no pueden ser adecuadamente representados por los hombres, las mismas tenían pocos escrúpulos a la hora de hablar "en nombre de" la mujer africana o hindú a la que nunca habían consultado (Pedersen 1991: 679). 
lonial, el consejo local de la ciudad keniana de Meru votó por unanimidad la prohibición de las intervenciones sobre los genitales femeninos. El consejo, formado por hombres, estaba bajo la administración colonial desde la década de 1930 (Thomas 2003: 79). La prohibición obtuvo como respuesta la circuncisión en masa de adolescentes que, sin ceremonias ni celebraciones, se circuncidaron a ellas mismas. Ngaitana fue el nombre que se dieron y formaron parte de la rebelión Mau Mau, trasformando la circuncisión en una medida para demostrar fidelidad a la tradición Kikuyu, el grupo étnico más grande de Kenia (Pedersen 1991: 677-680). La circuncisión fue adoptada como estrategia para reclamar su autonomía frente a los hombres del consejo local que trataban de controlar el cuerpo femenino y sobre todo frente al poder colonial que pretendía controlar la política africana.

Desafiando la dominación colonial, las intervenciones rituales sobre los genitales femeninos -interpretadas desde la perspectiva occidental como un mero instrumento de control y opresión de las mujeres - se trasformaron en un instrumento de empoderamiento y resistencia (Kershaw 1997: 190; Presley 1988). Hacer hincapié en la historia de la resistencia contra el poder colonial asociada a las intervenciones rituales sobre los genitales femeninos muestra la importancia de comprender las prácticas culturales como lugar de posibilidades múltiples, donde los individuos y los grupos activa y estratégicamente inventan la tradición y se reinventan a sí mismos (Njambi 2007: 705-706). Este enfoque permite un acercamiento a estas prácticas como un complejo conjunto de significados que están arraigados en una cultura transmitida de generación en generación y que al mismo tiempo varía en los distintos momentos políticos y contextos socio-culturales en los países subsaharianos, como también en la diáspora. Ngaitana testimonia cómo las intervenciones rituales sobre los genitales femeninos se convirtieron en un baluarte de resistencia contra los poderes coloniales y arroja una luz sobre cómo, tanto hoy como entonces, en el contexto migratorio, se pueden convertir en símbolos de las tradiciones, identidad cultural y autenticidad africana. Esto debería alertar a los legisladores occidentales sobre los riesgos de adoptar medidas de erradicación exclusivamente a través del derecho penal (La Barbera 2009).

\section{LA MEDICINA COMO "PROCESO DE CONTROL"}

Al considerar la "mutilación genital femenina" como violación de la integridad física se ha constreñido el cauce del discurso político internacional entre las categorías de los "derechos humanos", por un lado, y de la "medicina”, por el otro. Por tanto, el intento de acercarse a estas prácticas usando 
solo argumentos médicos y supuestamente neutrales invisibiliza sus inherentes aspectos socio-relacionales como si estos fueran meramente accesorios ${ }^{26}$. El uso de las categorías médicas occidentales como absolutas ignora además que también estas están culturalmente determinadas (Favretto y Mascherpa 1994: 164). Como se ha argumentado hasta aquí, las intervenciones rituales sobre los genitales femeninos son entendidas por las comunidades que las practican a través de los roles sociales, las relaciones de género y la construcción de la identidad étnica en que se arraigan.

Desde este punto de partida, pretendo cuestionar el uso de la medicalización como proceso legitimador a través de la noción de "proceso de control". Prestar atención a los procesos de control permite reconocer los mecanismos a través de los cuales individuos y grupos son influenciados, persuadidos a participar en su propia dominación y, de esta manera, controlados. Asimismo, el análisis de los procesos de control permite reconocer cómo se crean y funcionan los dogmas culturales, revelando la situacionalidad histórica, los mecanismos de producción y las fuerzas hegemónicas (Nader 1994: 1). La aplicación del concepto "proceso de control" al binomio salud/ patología en relación a las intervenciones rituales, estéticas y terapéuticas sobre los genitales femeninos ofrece una visión sugestiva sobre el poder normativo de la medicina. Utilizando este concepto analizo a continuación el caso emblemático de la cirugía intersexual, que constituye un ejemplo inequívoco de cómo el recurso a la medicina sirve para justificar intervenciones que, lejos de ser médicamente necesarias, son culturalmente requeridas.

Por cirugía intersexual se entiende la reasignación quirúrgica del sexo a niños con genitales ambiguos con el fin de ajustarlos a las expectativas sociales y evitarles — a ellos y sus padres - traumas psico-sociales, vergüenza y trastornos emocionales. Nancy Ehrenreich y Mark Barr reportan que los médicos prevén la operación de reasignación sexual para los niños que tienen el pene de tamaño inferior a la norma, a pesar del funcionamiento correcto de sus testículos. Aunque la eliminación de los testículos y la reducción del pene conlleve como efecto "colateral" la pérdida de la capacidad reproductiva y de satisfacción sexual, conseguir genitales aparentemente

\footnotetext{
${ }^{26}$ Los argumentos utilizados por muchas feministas occidentales en las campañas contra las intervenciones rituales sobre los genitales femeninos reflejan la dolorosa experiencia de la clitoridectomía victoriana. Desde luego, entre los diversos significados que conforman estas intervenciones rituales, las feministas occidentales consideran solo aquellos que están relacionados con el control de la sexualidad femenina y la virginidad, concibiendo todas las otras explicaciones simplemente como falsas y superpuestas. Desde esta perspectiva históricamente condicionada, las intervenciones rituales sobre los genitales femeninos son percibidas como un instrumento de las sociedades patriarcales dirigido únicamente a castrar y controlar la sexualidad femenina.
} 
normales es considerado médicamente prioritario. Además, las niñas que al nacer presentan el clítoris de un tamaño superior a la norma son sometidas a una transformación quirúrgica que les dota de un pene, aunque este nunca será funcional ${ }^{27}$. Es necesario además un tratamiento hormonal contínuo a lo largo de toda la vida para que la transformación sea eficaz.

Aunque se trate de una operación médicamente innecesaria que causa graves alteraciones físicas y psicológicas, la cirugía intersexual se realiza con frecuencia pero sigue siendo una gran desconocida. A pesar de las miles de operaciones realizadas cada año, los médicos no llevan a cabo ningún seguimiento a largo plazo de una operación técnicamente bien realizada (Kessler 1998: 53). Solo recientemente los estudios empíricos de la Intersexual Society of North America (ISNA) demuestran que los niños sometidos a la "normalización" de la cirugía intersexual a menudo experimentan problemas y traumas psicológicos, que van desde las depresiones graves hasta las inclinaciones suicidas ${ }^{28}$. Es interesante notar que si la cirugía intersexual pretende reparar o corregir una discapacidad, sin embargo, al transformar unos genitales funcionales, aunque anómalos, en órganos sexuales no fértiles y no sensitivos, termina por crear las condiciones intersexuales que pretende solucionar (Ehrenreich y Barr 2005: 109). La alteración quirúrgica y hormonal de tales deformidades es entendida como natural porque devuelve el cuerpo a lo que "debería haber sido", convirtiendo lo normal en normativo y lo normativo en natural (Kessler 1998: 24).

El caso de la cirugía intersexual arroja una luz distinta sobre las intervenciones rituales sobre los genitales femeninos ya que es un ejemplo elocuente de cómo el valor normativo del binomio salud/patología está estrechamente vinculado con la estrategia de legitimación o estigmatización de una práctica. La interpretación de la situación de patología y enfermedad no es solo un acto médico, sino social ya que los modelos interpretativos usados para definir una condición patológica están socialmente arraigados. Tales modelos poseen una eficacia normativa enorme en la medida en que

27 En 1967, en un hospital de EE.UU el mal funcionamiento de la máquina utilizada para la circuncisión causó la quemadura del pene de un niño de pocos meses llamado John. El Dr. John Money del Hospital Johns Hopkins sugirió trasformarlo quirúrgicamente en una niña. Durante 25 años, el caso conocido como John/Joan, fue considerado un triunfo de la medicina y, siguiendo este ejemplo, se realizaron miles de "reasignaciones sexuales infantiles". Solamente 30 años más tarde, el niño criado como una niña dijo la verdad: nunca se sintió una niña. Por esta razón, en edad más avanzada, se sometió a otra cirugía para cambiar el sexo y adoptó el nombre de David. David Reimer se suicidó en 2004. (Véase Colapinto 2000).

28 Véase Intersex Society of North America en www.isna.org [última consulta en fecha $01 / 05 / 2010]$. 
justifican el orden social que distingue lo "normal" de lo "patológico" (Foucault 1973).

\section{SOBRE LOS MECANISMOS DE PRODUCCIÓN DE LA "PATOLOGÍA"}

Carla Obermeyer destaca que entre las activistas que luchan para la eliminación de las intervenciones rituales sobre los genitales femeninos a menudo se comparte la actitud de asumir como verdades indiscutibles datos que en realidad solo en raras ocasiones son investigados (Obermeyer 1999: 97 y 2003: 404- 407). Incluso en ausencia de complicaciones, se asume que las intervenciones rituales sobre los genitales femeninos comprometen la salud. Como consecuencia, también el consentimiento de las mujeres adultas es valorado como irrelevante. En cambio, muchos estudios médicos indican que las complicaciones dependen en gran medida de las condiciones sanitarias inadecuadas en las que normalmente estos rituales se realizan (Shell-Duncan 2001: 1014).

Desde esta perspectiva, la solución de la hospitalización podría permitir evitar los riesgos para la salud, pero las activistas se oponen duramente en nombre del derecho a la satisfacción sexual que las mujeres "mutiladas" perderían para siempre. Sin embargo, algunos estudios recientes demuestran que incluso las mujeres a las que se le ha practicado el tipo de corte más drástico, o sea la infibulación, pueden tener una vida sexual satisfactoria (Lightfoot-Klein 1989: 80; Obermeyer 1999: 95 y Gruenbaum 2001: 133157). Según los datos recogidos por Lucrezia Catania y Omar Abdulcadir Hussen (2005 y 2007) utilizando el índice de función sexual femenina ${ }^{29}$ entre mujeres infibuladas — sin complicaciones sanitarias y con una relación emocionalmente satisfactoria con su pareja- solo el 3,65\% no consigue alcanzar el clímax (Catania y Abdulcadir 2005: 168-183; Catania 2007: 1670; Okonofua et al. 2002: 1092) $)^{30}$.

${ }^{29}$ El índice de función sexual femenina (Female Sexual Function Index, FSFI) es un instrumento válido y fiable para medir el funcionamiento sexual femenino. Se basa en un cuestionario que incluye datos sobre el deseo, la excitación, la lubricación, el orgasmo, la satisfacción y el dolor (Rosen et al. 2000).

30 Es interesante recordar que según importantes estudios ginecológicos la disfunción sexual femenina es un problema que afecta hasta un $40 \%$ de las mujeres con genitales íntegros en los EE.UU. (Rosen et al. 1993; Laumann et al. 1999). A propósito véase el artículo de Helen O'Connel sobre la anatomía del clítoris: "The entire cluster of related tissues (distal vagina, distal urethra and clitoris including the bulbs, crura, body and glans) should be included in the term clitoris. The distal vagina and urethra are clearly related, forming a midline core to the clitoris [...]. Such an inclusive concept would probably lead to the cessation of artificial discussions on the unnecessary separation of 
En esta perspectiva hay que considerar que la edad, el estado civil, el número de esposas cohabitantes en el hogar, el tiempo de permanencia en el extranjero, el grado de aculturación, el nivel educativo, la adhesión a los valores culturales de origen, así como el tipo de intervención genital, entre otros factores, pueden jugar un papel en la percepción de la propia sexualidad, imagen corporal y belleza ${ }^{31}$. En este sentido, Abulamini Oba argumenta que la campaña masiva contra las intervenciones rituales sobre los genitales femeninos puede tener efectos psicológicos negativos en las mujeres que se sometieron a la práctica antes de dejar su país de origen, sintiéndose mutiladas e incapaces de obtener satisfacción sexual. Este sentimiento puede dar lugar a una disfunción sexual, que a su vez, erróneamente es imputada a las intervenciones rituales sobres los genitales femeninos (Oba 2008: 12).

\section{A MODO DE CONCLUSIÓN}

A diferencia de la vaginoplastia y del implante de silicona, las intervenciones rituales sobre los genitales femeninos se persiguen como crímenes, sin considerar el consentimiento, las razones culturales que las fundamentan, las condiciones en las que se realizan, ni los reales perjuicios para la salud. Es preciso recordar que en el marco de las leyes actuales el consentimiento es necesario y suficiente para la realización de cualquier modificación del cuerpo femenino, excepto las que se realicen por razones rituales

the orgasmic focus, that is clitoral vs. vaginal" (O'Connell et al. 2005: 1194). Efectivamente, como afirma Thomas Laqueur, "The tale of the clitoris is a parable of culture, of how the body is forged into a shape valuable to civilization despite and not because of itself" (Laqueur 1990: 236).

31 Véase el reciente estudio de Lucrezia Catania y Omar Abdulcadir sobre un grupo de mujeres migrantes residentes in Italia que habían sido sometidas a una intervención ritual sobre los genitales femeninos en su país de origen. "Fifty-eight young ladies were living in Italy but were circumcised/infibulated in their country during childhood. As children in their own country, they experienced positive feelings about FGM/C, a sense of female completeness, they lived in a setting of social acceptance, felt family love and thought that FGM/C was 'something that testified beauty and courage'. Growing up in Western countries, their experience was transformed and given negative meanings: female mutilation, social stigma; they were depicted as victims of family violence and barbarity. Their sense of beauty changed into ugliness. The social stigmatization and the negative messages from the media regarding their 'permanently destroyed' sexuality provoke negative expectations on the possibility of experiencing sexual pleasure and provoke negative feelings about their own body image. The social criticism and the negative cultural meaning regarding their painful experience cause distortion of their cultural values and they undergo a sort of 'mental/psychological' infibulation which could result in iatrogenic sexual dysfunction" (Catania y Abdulcadir 2007: 1674; véase también Johansen 2007). 
(Allotey et al. 2001: 198). Sin embargo, la única diferencia parece consistir en que las primeras se realizan sobre mujeres blancas (léase "autónomas"), en clínicas y hospitales con el fin de mejorar el propio aspecto físico o la satisfacción sexual, en lugar de celebrar un ritual tradicional (del cual las mujeres son consideradas víctimas pasivas). De tal manera que las migrantes que se someten a estas intervenciones son consideradas desde el punto de vista jurídico como menores de edad e incapaces de decisiones autónomas (La Barbera 2009).

Tal incongruencia del sistema jurídico parece ser reveladora de la perspectiva etnocéntrica con la que se sigue juzgando al Otro como incapaz de auto-gobierno, víctima de una cultura retrógrada y necesitado de ser liberado por la civilización occidental. Las mujeres adultas migrantes originarias de países donde se practican las intervenciones rituales son y deben ser consideradas capaces de ejecutar elecciones libres y autónomas, al igual que las occidentales cuando deciden realizar el implante mamario de silicona o la vaginoplastia estética (Friedman 2003; Meyer 2001).

El análisis conjunto de las intervenciones rituales de los genitales femeninos con la vaginoplastia, el implante de mama y la cirugía intersexual demuestra claramente que en la interpretación de los conceptos de integridad física, salud y funcionamiento sexual como hechos biológicos se usan parámetros interpretativos diferentes dependiendo del contexto social, histórico y cultural. Por otro lado, resulta también evidente que estos parámetros están inmersos en un complejo conjunto de significados profundamente arraigados en la cultura y sujetos a los cambios de los distintos momentos políticos y contextos socio-culturales.

Encauzar el discurso sobre la mutilación genital femenina entre los derechos humanos y las (presuntamente neutrales) categorías médicas pone de manifiesto el resentimiento ideológico implicado en el discurso acerca de los derechos humanos. Considerar los derechos humanos como principios universales sin "declinarlos" en los distintos contextos culturales conduce a resultados no solo perversos, sino incluso contraproducentes. Un enfoque particularmente fecundo parece la utilización conjunta de la antropología y de la ciencia jurídica para comprender los instrumentos normativos como prácticas sociales, e investigar cómo podrían ser más eficaces para prevenir los conflictos entre las culturas que coexisten en la sociedad global.

\section{BIBLIOGRAFÍA CITADA}

Abdulcadir, O. 2006. "Research Center for Preventing and Curing FGM and Its Complications", en G. Denniston et al. (eds.), Bodily Integrity and the Politics of Circumcision. Culture, Controversy, and Change: 117-122. Nueva York: Springer. 
Abu-Sahlieh, S. 1994. "Islamic Law and the Issue of Male and Female Circumcision". Third World Legal Studies: 73-101.

Aixelá, Y. 2009. "El debate sobre las circuncisiones femeninas y su impacto en el marco jurídico, en los monoteísmos y en los feminismos en Egipto”, en J. Martí y Y. Aixelà (eds.), Desvelando el cuerpo: 287-298. Barcelona: CSIC.

Allotey, P. et al. 2001. "The Politics of Female Genital Surgery in Displaced Communities". Critical Public Health 11 (3): 189-201.

Althaus, F. 1997. "Female Circumcision: Rite of Passage or Violation of Rights?". International Family Planning Perspectives 23 (3): 130-133, http://www.guttmacher.org/pubs/ journals/2313097.pdf.

Bell, C. 1997. Ritual: Perspectives and Dimension. Oxford: Oxford University Press.

Bernardi, A.; B. Pastore y A. Pugiotto. 2008. Legalità penale e crisi del diritto oggi: un percorso interdisciplinare. Milán: Giuffre.

Blackledge, C. 2005. Historia de la Vagina. Barcelona: Península.

Boddy, J. 1982. "Womb as Oasis: The Symbolic Context of Pharaonic Circumcision in Rural Northern Sudan”. American Ethnologist 9 (4): 682-698.

Boyle, E. y S. Preves. 2000. "National Politics as International Process: The Case of AntiFemale Genital Cutting Laws". Law \& Society Review 34 (3): 703-737.

Catania, L. y O. Abdulcadir. 2005. Ferite per sempre. Le mutilazioni genitali femminili e la proposta del rito simbolico alternativo. Roma: Derive Approdi.

Catania, L., O. Abdulcadir et al. 2007. "Pleasure and Orgasm in Women with Female Genital Mutilation/Cutting (FGM/C)". Journal of Sexual Medicine 4: 1666-1678.

Chambers, C. 2004. "Are Breast Implants Better Than Female Genital Mutilation? Autonomy Gender Equality and Nussbaum's Political Liberalism". Critical Review of International Social and Political Philosophy 7 (4): 1-33.

Ciminelli, M. 2002. "Le ragioni culturali delle mutilazioni genitali femminili: note critiche sulla definizione di Mgf dell'Oms/Who”. La Ricerca Folklorica 46: 39-50.

Chase, C. 2002. "Cultural Practice or Reconstructive Surgery? US Genital Cutting, the Intersex Movement, and Medical Double Standards", en S. James y C. Robertson (eds.), Genital Cutting and Transnational Sisterbood. Disputing US Polemics: 126-151. Chicago: University of Illinois Press.

Coco, L. 1994. "Silicone Breast Implants in America: A Choice of the 'Official Breast'?". Kroeber Anthropological Society Papers 77: 103-132.

Colapinto, J. 2000. As Nature Made Him: The Boy Who was Raised as a Girl. Nueva York: Harper Collins.

Davis, K. 2003. "Surgical Passing: Or Why Michael Jackson's Nose Makes Us Uneasy". Feminist Theory 4 (1): 73-92.

Davis-Floyd, R. 2008. "Ritual", en W. Darity (ed.), International Encyclopedia of Social Sciences, vol. 7. Nueva York: Macmillan.

Ehrenreich, N. y M. Barr. 2005. "Intersex Surgery, Female Genital Cutting, and Selective Condemnation of Cultural Practices". Harvard Civil Rights-Civil Law Review 40: 71140 .

El Safti, M. 2007. "Mujeres egipcias en perspectiva ¿Derechos o práctica?”. Quaderns de la Mediterrània 7: 143-148, http://www.iemed.org/publicacions/quaderns/7/ e143_ElSafty.pdf.

El-Tom, A. 1998. "Female circumcision and ethnic identification in Sudan with special reference to the Berti of Darfur". GeoJournal 46: 163-170. 
Erlich, M. 1986. La femme blessée: Essai sur les mutilations sexuelles féminines. París: l'Harmattan.

Favretto, A. y F. Mascherpa. 1994. "Le concezioni di salute e di malattia nell'interazione terapeutica interculturale". Dei delitti e delle pene 1: 148-149.

Foucault, M. 1973. The Birth of Clinic: An Archeology of Medical Perception. Londres: Tavistock.

Freud, S. 1925. "Some Psychical Consequences of the Anatomical Distinction between the Sexes", en The Standard Edition of the Collected Work of Sigmund Freud, vol. XIX: 241-258. Londres: Hogarth Press.

Friedman, M. 2003. Autonomy, Gender, Politics. Oxford: Oxford University Press.

Fusaschi, M. 2003. I segni sul corpo. Per un'antropologia delle modificazioni dei genitali femminili. Turín: Bollati Boringhieri.

Galeotti, E. 2007. "Relativism, Universalism, and Applied Ethics: The Case of Female Circumcision”. Constellations 14 (1): 91-111.

Grande, E. 2004. "Hegemonic Human Rights and African Resistance: Female Circumcision in a Broader Comparative Perspective". Global Jurist Frontiers 4 (2): 1-21.

Grassivaro Gallo, P.; E. Tita y F. Viviani. 2006. "At the Roots of Ethnic Female Genital Modification: Preliminary Report”, en G. Denniston; P. Grassivaro Gallo, et al. (eds.), Bodily Integrity and the Politics of Circumcision: Culture, Controversy, and Change: 49-55. Nueva York: Springer.

Gruenbaum, E. 2001. The Female Circumcision Controversy: An Anthropological Perspective. Filadelfia: University of Pennsylvania Press.

Guiné, A. y F. Moreno Fuentes. 2007. "Engendering Redistribution, Recognition, and Representation: The Case of Female Genital Mutilation (FGM) in the United Kingdom and France". Politics \& Society 35 (3): 477-519.

Hart, M.; J. Stevens y F. Lieberman. 1990. Drumming at the Edge of Magic: A Journey into the Spirit of Percussion. San Francisco, CA: Harper.

Hernlund, Y. 2000. "Cutting Without Ritual and Ritual Without Cutting: Female Circumcision and 'Re-ritualization' of initiation in the Gambia", en B. Shell-Duncan e Y. Hernlund (eds.), Female Circumcision in Africa: Culture, Controversy and Change: 235252. Boulder (Chicago): Lynne Rienner Publishers.

Johansen, E. 2007. "Experiencing Sex in Exile. Can Genitals Change Their Gender? On Conceptions and Experiences Related to Female Genital Cutting (FGC) Among Somalis in Norway", en B. Shell- Duncan e Y. Hernlund (eds), Transcultural Bodies. Female Genital Cutting in Global Context: 248-277. New Brunswick (NJ): Rutgers University Press.

Kelly, J. y M. Kaplan. 1990. "History, Structure, and Ritual”. Annual Review of Anthropology 19: $119-150$.

Kenyatta, J. 1938. Facing Mount Kenya. The Traditional Life of the Giyuku. Londres: Secker and Warburg.

Kershaw, G. 1997. Mau Mau from Below. Athens (OH): Ohio University Press.

Kerzter, D. 1988. Ritual, Politics, and Power. New Haven: Yale University Press.

Kessler, S. 1998. Lessons from the Intersexed. New Brunswick (NJ): Rutgers University Press.

La Barbera, MC. 2009. "Revisiting the anti-Female Genital Mutilation Feminist Discourse". Diritto \& Questioni Pubbliche 9: 485-507, http://www.dirittoequestionipubbliche.org/ page/2009_n9/05_studi-03_MC_LaBarbera.pdf.

La Barbera, MC. 2010. "Mujeres, minorías y derecho penal. El trato jurídico de la "mutilación genital femenina' en los países europeos”. Sortuz: Oñati Journal of Emergent Socio-legal Studies 4 (1): 34-67. 
La Barbera MC. y C. Cortina 2010. "La legislazione sulla 'mutilazione genitale femminile' in Europa". Neodemos, http://www.neodemos.it/index.php?file=onenews\&form_ id_notizia $=411$.

Laqueur, T. 1990. Making Sex: Body and Gender from the Greeks to Freud. Cambridge (Mass.): Harvard University Press.

Laumann, E. et al. 1999. "Sexual dysfunction in the United States: prevalence and predictors". Journal of the American Medical Association 281 (6): 537-544.

Lightfoot-Klein, H. 1989. Prisoners of Ritual. An Odyssey into Female Genital Circumcision in Africa. Nueva York: Harrington Park Press.

Little, K. 1949. "The Role of the Secret Society in Cultural Specialization". American Anthropologist 51 (2): 199-212.

Masters, W. et al. 1995. Human Sexuality. Nueva York: Harper Collins.

Meyers, D. 2000. "Feminism and Women's Autonomy: The Challenge of Female Genital Cutting". Metaphilosophy 31(5): 469-91.

Mitchel, J. 1996. "Ritual", en A. Barnard y J. Spencer (eds.), Encyclopedia of Social and Cultural Anthropology: 490-493. Londres: Routledge.

Moore, S. (ed.). 2005. Law and Anthropology. Oxford: Blackwell.

Moore, S. y B. Myerhoff. (eds). 1977. Secular Ritual. Assen: Van Gorcum.

Morinis, A. 1985. "The Ritual Experience: Pain and the Transformation of Consciousness in Ordeals of Initiation". Ethos 13 (2): 150-174.

Nader, L. 1994. "Controlling Processes". Kroeber Anthropological Society Papers 77: 1-9.

Njambi, N. 2007. "Irua Ria Atumia and Anti-Colonial Struggles Among the Gikuyu Of Kenya: A Counter Narrative on Female Genital Mutilation". Critical Sociology 33 (4): 689-708.

Nnaemeka, O. 2001. "If Female Circumcision Did Not Exist, Western Feminism Would Invent It", en S. Perry y C. Schenk (eds.), Eye to Eye. Women Practicing Development across Cultures: 171-189. Londres: Zed Book.

Nussbaum, M. 1999. Sex and Social Justice. Oxford: Oxford University Press.

O'Connell, H.; K. Sanjeevan y J. Hutson. 2005. "Anatomy of the Clitoris". The Journal of Urology 174: 1189-1195.

Oba, A. 2008. "Female Circumcision as Female Genital Mutilation: Human Rights or Cultural Imperialism?". Global Jurist 8 (3): 1-38.

Obermeyer, C. 1999. "Female Genital Surgery: The Known, the Unknown, the Unknowable". Medical Anthropology Quarterly 13 (1): 79-106.

Obermeyer, C. 2003. "The Health Consequences of Female Circumcision: Science, Advocacy, and Standard of Evidence". Medical Anthropology Quarterly 17 (3): 394412 .

Obermeyer, C. 2005. "The Consequences of Female Circumcision for Health and Sexuality: An Update on the Evidence". Culture, Health \& Sexuality 7 (5): 444-461.

Okonofua, F. et al. 2002. "Association between Female Genital Cutting and Correlates of Sexual and Gynaecological Morbidity in Edo State, Nigeria”. BJOG: An International Journal of Obstetrics and Gynaecology 109: 1089-1096.

Pasquinelli, C. 2007. Infibulazione: Il corpo violato. Roma: Meltemi.

Pedersen, S. 1991. "National Bodies, Unspeakable Acts: The Sexual Politics of Colonial Policy-making". The Journal of Modern History 63 (4): 647-680.

Presley, C. 1988. "The Mau Mau Rebellion, Kikuyu Women, and Social Change". Canadian Journal of African Studies/Revue Canadienne des Études Africaines 22 (3): 502-527. 
Rappaport, R. 1999. Ritual and Religion in the Making of Humanity. Cambridge: Cambridge University Press.

Rosen, R. et al. 1993. "Prevalence of Sexual Dysfunction on Women: Results of a Survey Study of 329 Women in an Outpatient Gynecological Clinic". Journal of Sex and Marital Therapy 19 (3): 171-188.

Rosen, R. et al. 2000. "The Female Sexual Function Index (FSFI): A Multidimensional SelfReport Instrument for the Assessment of Female Sexual Function". Journal of Sex \& Marital Therapy 26 (2): 191-208.

Sheehan, E. 1981. "Victorian Clitoridectomy: Isaac Baker Brown and His Harmless Operative Procedure". Medical Anthropology Newsletter 12 (4): 9-15.

Sheldon, S. y S. Wilkinson. 1998. "Female Genital Mutilation and Cosmetic Surgery: Regulating Non-Therapeutic Body Modification". Bioethics 12 (4): 263-285.

Shell-Duncan, B. 2001. "The Medicalization of Female 'Circumcision': Harm Reduction or Promotion of a Dangerous Practice?". Social Science \& Medicine 52: 1013-1028.

Shweder, R. 2002. "What about Female Genital Mutilation? And Why Understanding Culture Matters in the First Place", en R. Shweder et al. (eds.), Engaging Cultural Differences: 216-251. Nueva York: Russel Sage Foundation.

Spivak, C. 1988. "Can The Subaltern Speak? “, en C. Nelson y L. Grossberg (eds.), Marxism and the interpretation of Culture: 271-313. Chicago: University of Illinois Press.

Thiam, A. 1986. Speak Out, Black Sisters: Feminism and Oppression in Black Africa. London: Pluto Press.

Thomas, L. 2003. Politics of the Womb Women, Reproduction, and the State in Kenya. Berkeley: University of California Press.

Turner, V. 1967. The Forest of Symbols: Aspects of Ndembu Rituals. Ithaca (NY): Cornell University Press.

Van Howe, R. 1997. "Why Does Neonatal Circumcision Persist in the United States?", en G. Denniston y M. Milos (eds.), Sexual Mutilations. A Human Tragedy: 111-119. Nueva York: Plenum Press.

Walley, C. 1997. "Searching for 'voices': Feminism, Anthropology, and the Global Debate over Female Genital Operations". Cultural Anthropology 12 (3): 405-438.

Warner, L. 1959. The Living and the Dead: A Study of the Symbolic Life of Americans. New Haven (CT): Yale University Press.

Wasunna, A. 2000. "Towards Redirecting the Female Circumcision Debate: Legal, Ethical and Cultural Considerations". McGill Journal of Medicine 5 (2): 104-110.

Weil-Curiel, L. 2001. "Female Genital Mutilation in France: A Crime Punishable by Law", en S. Perry y C. Schenck (eds.), Eye to Eye. Women Practicing Development across Cultures: 190-197. Londres: Zed Book.

Fecha de recepción: 12 de julio de 2009

Fecha de aceptación: 31 de mayo de 2010 